\title{
Diurnal Salivary Cortisol in Sarcopenic Postmenopausal Women: The OsteoLaus Cohort
}

\author{
Elena Gonzalez Rodriguez ${ }^{1,7}(1) \cdot$ Pedro Marques-Vidal ${ }^{2}\left({ }^{\infty} \cdot\right.$ Bérengère Aubry-Rozier $^{3} \cdot$ Georgios Papadakis $^{4}$.

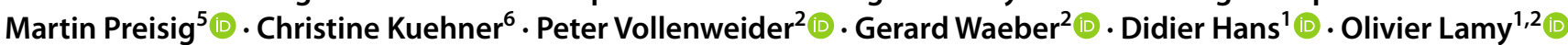

Received: 15 February 2021 / Accepted: 30 April 2021 / Published online: 18 May 2021

(c) The Author(s) 2021

\begin{abstract}
Sarcopenia, similar to hypercortisolism, is characterized by loss of muscle mass and strength. Cortisol circadian rhythm changes with aging (blunted late-day nadir values) were suggested to contribute to this decline. We aimed to explore the relationship between diurnal salivary cortisol values and sarcopenia diagnosis and its components in postmenopausal women. This is a cross-sectional study within the OsteoLaus population-based cohort in Lausanne (Switzerland). Participants had a body composition assessment by dual X-ray absorptiometry (DXA), a grip strength (GS) measure, and salivary cortisol measures (at awakening, 30 min thereafter, $11 \mathrm{AM}$ (sc-11 AM) and 8 PM (sc-8PM)). Associations between salivary cortisol and sarcopenia diagnosed by six different criteria (based on appendicular lean mass (ALM) assessed by DXA, and muscle strength by GS), and its components, were analyzed. 471 women aged $>50$ years $(63.0 \pm 7.5)$ were included. Various definitions identified different participants as sarcopenic, who consistently presented higher salivary cortisol at $11 \mathrm{AM}$ and/or 8 PM. There were no associations between salivary cortisol levels and ALM measures, either absolute or after correction to height squared (ALM index) or body mass index. GS was inversely correlated to sc-11AM $(r=-0.153, p<0.001)$ and sc8PM $(r=-0.118, p=0.002)$. Each $10 \mathrm{nmol} / \mathrm{l}$ increase of sc-11AM, respectively sc-8PM, was associated with a GS decrease of 1.758 (SE 0.472 ) kg, respectively 2.929 (SE 1.115) kg. In postmenopausal women, sarcopenia is associated with higher salivary cortisol levels at $11 \mathrm{AM}$ and $8 \mathrm{PM}$. An increase of daily free cortisol levels in the physiological range could participate to sarcopenia development by decreasing muscle function in postmenopausal women.
\end{abstract}

Keywords Salivary cortisol $\cdot$ Sarcopenia $\cdot$ Body composition $\cdot$ OsteoLaus $\cdot$ Cohort

Elena Gonzalez Rodriguez

elena.gonzalez-rodriguez@chuv.ch

1 Interdisciplinary Center for Bone Diseases, Service of Rhumatology, Lausanne University Hospital and University of Lausanne, Lausanne, Switzerland

2 Service of Internal Medicine, Department of Medicine, Lausanne University Hospital and University of Lausanne, Lausanne, Switzerland

3 Service of Genetic Medicine, Department of Medicine, Lausanne University Hospital and University of Lausanne, Lausanne, Switzerland

4 Service of Endocrinology, Diabetology and Metabolism, Department of Medicine, Lausanne University Hospital and University of Lausanne, Lausanne, Switzerland
5 Epidemiology and Psychopathology Research Unit, Department of Psychiatrics, Lausanne University Hospital and University of Lausanne, Lausanne, Switzerland

6 Research Group Longitudinal and Intervention Research, Department of Psychiatry and Psychotherapy, Central Institute of Mental Health, Medical Faculty Mannheim, Heidelberg University, Heidelberg, Germany

7 Interdisciplinary Center of Bone Diseases, Service of Rheumatology, CHUV, Lausanne University Hospital, Rue Pierre-Decker 4, 1011 Lausanne, Switzerland 


\section{Introduction}

Sarcopenia, characterized by the loss of skeletal muscle mass and strength, is a major risk factor for physical frailty, poor health-related quality of life, and premature death in older people [1], as well as a negative prognostic factor in numerous diseases [2]. Its prevalence ranges from 1 to $29 \%$ in community-dwelling populations [3]. Different definitions of sarcopenia have been proposed using different methods or cut-off points for assessing muscle mass and function [4], which lead to very large differences in sarcopenia prevalence in a given population [5] and even classify different individuals as sarcopenic [6]. Many different complex and not yet fully understood pathophysiological mechanisms may be implicated in its development [7].

Overt hypercortisolism leads to muscle atrophy and weakness [8]. With aging, changes in the cortisol circadian rhythm induce long-term exposure to mildly higher cortisol levels, due to higher late-day and evening nadir values [9, 10]. These can be observed in salivary cortisol, an easy and non-stressful method of cortisol measurement [11]. It has higher clinical utility, because of the better convenience than other sampling methods (can be performed in the outpatient setting), and because it represents unbound active cortisol levels.

Modifications of the hypothalamic-pituitary-adrenocortical axis with aging have been suggested to contribute to the decline in lean body mass [12], although data are scarce and heterogeneous. Patients with mild hypercortisolism in the context of an adrenal incidentaloma have either lower lean mass as measured by bioimpedance [13] or lower body mass index (BMI)-adjusted lean mass assessed by dual-Xray absorptiometry (DXA) [14]. Previous studies have suggested that higher diurnal or evening salivary cortisol, or blunted cortisol circadian rhythm, are associated with low muscle function measured by different methods [15-18]. Only one small study $(n=45)$ [19] indicated a relationship between sarcopenia diagnosis and blunted diurnal variation of cortisol.

In this context, we aimed to determine whether cortisol levels at different time-points, or the area under the curve, were associated with sarcopenia, taking into account definitions including different diagnostic cut-offs for lean mass by DXA, with or without muscle function measured by peak grip strength (GS).

\section{Materials and Methods}

\section{Setting}

We analyzed data from the previously described OsteoLaus sub-study of the CoLausIPsyCoLaus study [20]. Briefly, CoLaus|PsyCoLaus is an ongoing prospective cohort study on cardiovascular and mental diseases determinants in a population-based sample of 6738 randomly selected individuals from Lausanne, Switzerland [21]. The OsteoLaus sub-study aims to compare different models of fracture risk prediction and to assess the relationship between osteoporosis and cardiovascular diseases [22]. OsteoLaus invited all women aged 50-80 years within 6 months of first follow-up of the CoLausIPsyCoLaus study (September 2009-December 2012) to have a DXA scan; $85 \%$ accepted [22]. Also, at first follow-up CoLaus|PsyCoLaus participants aged $>50$ benefited of GS measures in the context of a frailty substudy, and of an assessment of the corticotropic axis by measures of salivary cortisol circadian rhythm [23] (Supplementary Fig. 1).

The institutional Ethics Committee of the University of Lausanne, later the Ethics Commission of Canton Vaud (www.cer-vd.ch), approved the baseline and first followup of CoLaus study (references 16/03 and 33/09), and the OsteoLaus study (reference 215-09). All participants signed a written informed consent after having received a detailed description of the goal and funding of the studies. Human studies are in accordance with the ethical standards of the 1964 Declaration of Helsinki and its later amendments.

\section{Participants}

All participants were included except if they presented the previously defined exclusion criteria [20] or lacked data. Briefly, participants were excluded if they did not participate to the first CoLaus|PsyColaus follow-up, had no salivary cortisol measure, or lacked grip strength (GS) measurement, as well as if they were current smokers or treated with systemic glucocorticoids $\geq 3$ months. As in our previous study [20], salivary cortisol values $>4$ standard deviations (SD) higher than the mean were considered as outlier values and excluded.

\section{Salivary Cortisol Measures}

At the end of the interview of the first psychiatric follow-up assessment [24] participants received little swabs (Salivettes, Sarstedt, Sevelen, Switzerland) for salivary cortisol collection on a working day at awakening, 30 min thereafter, at 11 AM (sc-11AM), and at 8 PM (sc-8PM). Subjects were 
instructed not to brush their teeth and to refrain from eating, drinking, smoking, and exercising $30 \mathrm{~min}$ prior to and during the sampling procedure [25]. Saliva samples were stored at patient's freezers and then at $-20{ }^{\circ} \mathrm{C}$ at the laboratory until biochemical analysis. Free cortisol levels were measured using a commercially available chemiluminescence assay (IBL, Hamburg, Germany). Inter- and intra-assay coefficients of variability were $<9 \%$.

Diurnal Area Under the Curve values (sc-AUC) were calculated using the AUCground trapezoid method as performed by Pruessner et al. [26], and Cortisol Awakening Response (CAR) by subtracting the salivary cortisol awakening value from the $30 \mathrm{~min}$ after awakening value.

\section{Anthropometric Measures and Body Composition Assessment}

All participants had their height measured using the same portable stadiometer (Seca version 216, Seca, Chino, CA, USA) with precision $0.1 \mathrm{~cm}$ and body weight with the same electronic scale (Seca Clara 803, Seca, Chino, CA, USA) with a precision of $0.1 \mathrm{~kg}$, with the participant barefoot and in minimum clothing. BMI was calculated by dividing the individual's weight by height squared $\left(\mathrm{kg} / \mathrm{m}^{2}\right)$.

Body composition assessment was done using the Discovery A System (Hologic, Waltham, MA, USA), in accordance with published guidelines by the International Society for Clinical Densitometry [27]. Participants were placed centered on the scanning field in a supine position, with palms down and arms at sides, slightly separated from the trunk. Regions of interest (ROIs) were defined by the analytical program and included total body, trunk, head, pelvis, upper limbs, and lower limbs. For each region, DXA scanned weight of total, fat, and lean body mass. For the actual study, three lean mass measures were used: appendicular lean mass (ALM), calculated by the addition of the four limbs lean mass; ALM index (ALMI), computed as the ratio of ALM over height squared; and ALM divided by body mass index (ALM/BMI).

\section{Grip Strength (GS)}

GS was assessed with a Baseline ${ }^{\circledR}$ hydraulic hand dynamometer (Fabrication Enterprises, Inc., White Plains, NY, USA), in the morning. According to the American Society of Hand Therapists guidelines [28], subjects were seated, shoulders adducted and neutrally rotated, elbow flexed at $90^{\circ}$, forearm in neutral position, and wrist between $0^{\circ}$ and $30^{\circ}$ of dorsiflexion. The highest value (expressed in kilograms) of three consecutive measurements with the selfreported dominant hand was used for the analysis.

\section{Sarcopenia Diagnosis}

Classifications defined in Caucasian participants with cutoff values for lean mass measured by DXA and for muscle strength measured by GS were used (Table 1). We first classified participants based on the two current definitions. FNIH (Foundation for the National Institutes of Health Sarcopenia Project) sarcopenia diagnosis criteria (FNIH2014) identifies "severe sarcopenia" with ALM either in absolute values or adjusted to BMI [29]. The revised consensus definition of the European Working Group on Sarcopenia in Older People (EWGSOP2) [1] defines sarcopenia as "probable" in case of low GS, and "confirmed" if participants have both low GS and low muscle mass, either in absolute (ALM) or height-adjusted values (ALMI). Probably sarcopenic participants by EWGSOP 2 criteria considers only low GS, they
Table 1 Cut-off values of grip strength and lean mass assessed by DXA for sarcopenia diagnosis and classification in women, depending on the defined criteria

\begin{tabular}{lllllc}
\hline & Grip strength & & & Lean mass \\
\cline { 2 - 3 } \cline { 5 - 6 } \cline { 5 - 6 } & Measure & Cut-point & & Measure & Cut-point \\
\hline FNIH 2014 [29] & Peak value $(\mathrm{kg})$ & $<16.00$ & & ALM $(\mathrm{kg})$ & 15.02 \\
& & & & ALM/BMI $\left(\mathrm{kg} /\left(\mathrm{kg} / \mathrm{m}^{2}\right)\right)$ & 0.512 \\
EWGSOP2, 2019 [1] & Peak value $(\mathrm{kg})$ & $<16.00$ & & ALM $(\mathrm{kg})$ & 15.00 \\
& & & & ALMI $\left(\mathrm{kg} / \mathrm{m}^{2}\right)$ & 5.5 \\
FNIH 2017 [30] & Peak value $(\mathrm{kg})$ & $<19.99$ & & ALM $(\mathrm{kg})$ & 14.12 \\
& & & & ALM $/ \mathrm{BMI}\left(\mathrm{kg} /\left(\mathrm{kg} / \mathrm{m}^{2}\right)\right)$ & 0.591 \\
EWGSOP, 2010 [31] & Peak value $(\mathrm{kg})$ & $<20.00$ & & ALMI $\left(\mathrm{kg} / \mathrm{m}^{2}\right)$ & 5.5 \\
\hline
\end{tabular}

ALM appendicular lean mass, BMI body mass index, ALMI ALM/height ${ }^{2}$, FNIH Foundation for the National Institutes of Health Sarcopenia Project; sarcopenia is defined by the presence of both low grip strength and low lean mass. 2014 criteria would define "severe sarcopenia", while 2017 criteria would define "mild to moderate sarcopenia", EWGSOP2 European Working Group on Sarcopenia in Older People, revised consensus; sarcopenia is defined as "probable" in the presence of only low grip strength, and "confirmed" in the presence of both low grip strength and low lean mass, EWGSOP European Working Group on Sarcopenia in Older People; sarcopenia is defined by the presence of both low grip strength and low lean mass 
are thus not comparable to sarcopenic ones, which include low muscle mass, so they were added to the non-sarcopenic. Due to close or identical cut-off values for ALM in absolute values and GS, FNIH2014 and EWGSOP2 identified the same participants as sarcopenic and were considered together. Two other alternative definitions have also been applied: One using less stringent cut-offs to identify "mild to moderate sarcopenia" derived from part of the population of the original FNIH study (FNIH2017) [30] and the definition of the first EWGSOP consensus [31] in which participants are classified as sarcopenic in the presence of both low GS and low ALMI

\section{Statistical Analysis}

Parameters were compared after classification of participants: (i) following different sarcopenia diagnosis criteria, (ii) by tertiles of sc-11AM and sc-8PM, and (iii) by tertiles of age.

Statistical analyses were conducted using Stata v16.1 ${ }^{\odot}$ (StataCorp, College Station, TX, USA) for Windows ${ }^{\circledR}$. Due to their skewed distributions, salivary cortisol values were log-transformed to approach a normal distribution. Descriptive results were expressed as number of participants (percentage) for categorical variables and as average \pm standard deviation for continuous variables.

Between-group comparisons were performed using chisquare or Fisher's exact test for categorical variables and Kruskal-Wallis test, student's $t$-test, or analysis of variance for continuous variables. For continuous variables, multivariable analysis was conducted using analysis of variance adjusting for age and $\mathrm{BMI}$; results were expressed as adjusted mean \pm standard error. Associations between salivary cortisol markers and sarcopenia components were assessed using (1) Spearman correlation and (2) linear regression using sarcopenia components as dependent and cortisol markers as independent variables; results were expressed as slope, namely the regression coefficient, (standard error) for an increment of $10 \mathrm{nmol} / \mathrm{l}$ salivary cortisol. Statistical significance was considered for a two-sided test with $p$-value $<0.05$.

\section{Results}

\section{Selection of Participants}

Of the initial 1475 participants from OsteoLaus, 705 (47.8\%) had both cortisol and GS measures (Fig. 1 and Supplementary Fig. 1) and 471 (31.9\%) had cortisol, GS, and body composition measures (Fig. 1). Supplementary Table 1 summarizes the characteristics of included and excluded participants according to sarcopenia diagnosis. Included

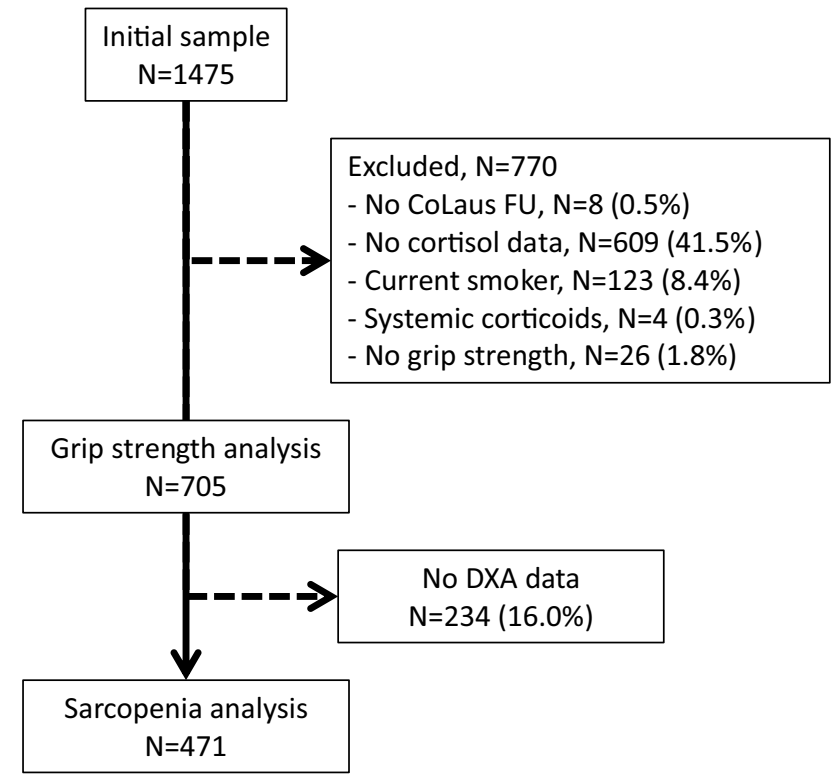

Fig. 1 Flowchart of the study

participants were younger, had higher GS measures, and lower sc-8PM levels.

\section{Participant's Classification Using Different Criteria for Sarcopenia Diagnosis}

Participants were classified as sarcopenic or non-sarcopenic depending on the cut-offs values in Table 1. As only EWGSOP 2 criteria define probably sarcopenic by low GS alone, those were added to non-sarcopenic for the analysis. Probably sarcopenic by EWGSOP2-ALM $(n=21)$ were heavier (weight: $74.7 \pm 12.3$ vs. $67.7 \pm 12.1 \mathrm{~kg}, p=0.04$; and BMI: $28.6 \pm 4.7$ vs. $\left.25.9 \pm 4.5 \mathrm{~kg} / \mathrm{m}^{2}, p=0.02\right)$; and probably sarcopenic by EWGSOP2-ALMI $(n=33)$ were older $(66.6 \pm 7.4$ vs. $62.6 \pm 7.8$ years, $p=0.012)$, shorter $(158.6 \pm 6.6$ vs. $162.0 \pm 6.7 \mathrm{~cm}, p=0.02)$, and had lower ALM/BMI $\left(0.62 \pm 0.10\right.$ vs. $\left.0.68 \pm 0.10 \mathrm{~kg} /\left(\mathrm{kg} / \mathrm{m}^{2}\right), p=0.004\right)$, when compared with non-sarcopenic. There was no difference in any other measured parameter or any cortisol value (results not shown).

The number of identified sarcopenic individuals is different depending on the criteria used, with almost no overlap, from $n=5$ (1.1\%) diagnosed by FNIH2014-ALM/BMI to $n=31(6.6 \%)$ using the ALM/BMI cut-off. This resulted in a total of 50 sarcopenic participants when applying all definitions $(10.6 \%)$ and 22 when applying the current definitions (Fig. 2); no participant is considered sarcopenic by all the 6 definitions (central gray square).

Characteristics of the participants are shown in Table $2 \mathrm{a}$ for current sarcopenia definitions and in Supplementary Table 2a for the alternative definitions. Participants 
Fig. 2 Number of participants diagnosed as sarcopenic depending on the current definitions, and their association with higher salivary cortisol at 11 AM, 20 PM, or both

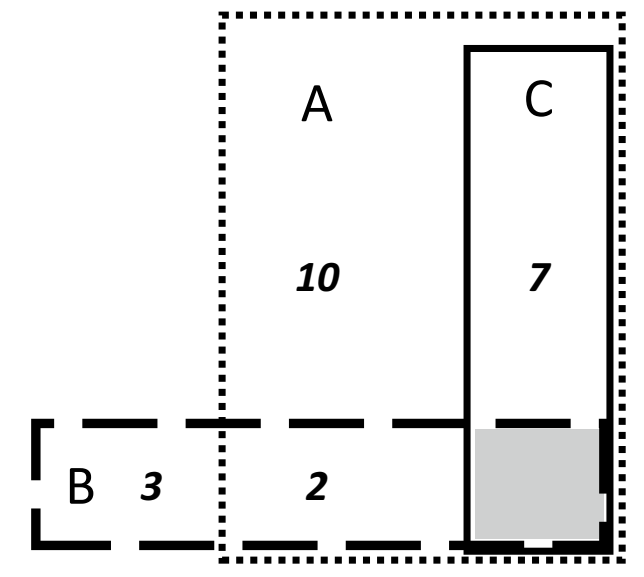

\footnotetext{
A: EWGSOP2 \& FNIH2014 - ALM, higher 11 AM and 20 PM salivary cortisol $(n=19)$

$\underline{B} 1$

FNIH2014 - ALM/BMI, higher 20 PM salivary cortisol ( $n=5)$

C EWGSOP2 - ALMI, higher 11 AM salivary cortisol $(n=7)$

Sarcopenia according all criteria $(n=0)$
}

identified as sarcopenic were older compared to non-sarcopenic across definitions. There were more sarcopenic participants in higher age tertiles (Supplementary Table 3), with a prevalence going from 0.0 to $1.9 \%$ in tertile 1 (50.3-59.0 years) to $1.9-12.1 \%$ in tertile 3 (66.5-80.8 years). Older participants had lower values of GS and ALM, either in absolute values or corrected to BMI or height (ALMI). Salivary cortisol values at $11 \mathrm{AM}$ are significantly higher with age, with a similar trend for salivary cortisol values at $8 \mathrm{PM}$ (Supplementary Table 3).

Anthropometric characteristics of participants identified as sarcopenic are different depending on the definition (Table 2a and Supplementary Table 2a). BMI is lower in sarcopenic than in non-sarcopenic participants for definitions using ALM or ALMI cut-offs, while it is higher as expected by definition when using ALM/BMI cut-offs. Moreover, when using ALM/BMI cut-offs ALMI is not different between sarcopenic and non-sarcopenic participants, and conversely when using ALMI cut-offs ALM/BMI is similar in both groups. Yet both are statistically lower in sarcopenic participants when absolute ALM cut-offs are used.

\section{Salivary Cortisol Values in Sarcopenic Participants}

There was no difference in salivary cortisol between sarcopenic and non-sarcopenic participants at awakening, $30 \mathrm{~min}$ later (Table $2 \mathrm{~b}$ and Supplementary Table $2 b$ ), or the CAR (data not shown). Sc-11AM was significantly higher in sarcopenic participants according to FNIH2014\&EWGSOP2-ALM, EWGSOP2-ALMI, and EWGSOP-ALMI both in bivariate and multivariate analyses. Sc-8PM was significantly higher in sarcopenic participants according to FNIH2014\&EWGSOP2-ALM, FNIH2014ALM/BMI, and EWGSOP-ALMI both in bivariate and multivariate analyses. sc-AUC was higher in sarcopenic participants according to FNIH2014\&EWGSOP2-ALM, EWGSOP2-ALMI, FNIH2017-ALM/BMI, and EWGSOPALMI, both in bivariate and multivariate analyses. When participants considered sarcopenic by any definition were analyzed, the three sc-11AM, sc-8PM, and sc-AUC were higher in sarcopenic participants in bivariate and multivariate analyses (Table 3). Figure 2 graphically shows which current sarcopenia definition is associated with higher sc11AM, higher sc-8PM, or both.

\section{Participant's Classification According to sc-11 AM and sc-8PM Tertiles}

Participants with higher sc-11AM (Table 4) were older, with a similar trend for those with higher sc-8PM (Table 4). There was no difference in any anthropometric or body composition parameter (weight, height, BMI, ALM, ALM/BMI, or ALMI) between cortisol tertiles. With increasing sc-11AM or sc-8PM, GS was lower, and there were more sarcopenic participants for all definitions except for FNIH2014-ALM/BMI (sc-11AM and sc-8PM) and FNIH2017-ALM (sc-11AM). These differences 
Table 2 Classification of participants according to current criteria for sarcopenia diagnosis

\begin{tabular}{|c|c|c|c|c|c|c|c|c|c|}
\hline & \multicolumn{3}{|c|}{ FNIH 2014 \& EWGSOP2-ALM } & \multicolumn{3}{|c|}{ FNIH 2014-ALM/BMI } & \multicolumn{3}{|c|}{ EWGSOP2-ALMI } \\
\hline & None & Sarcopenic & $p$-value* & None & Sarcopenic & $p$-value* & None & Sarcopenic & $p$-value* \\
\hline \multicolumn{10}{|c|}{ (a) Participants' characteristics } \\
\hline$N(\%)$ & $452(96.0)$ & $19(4.0)$ & & $466(98.9)$ & $5(1.1)$ & & $464(98.5)$ & $7(1.5)$ & \\
\hline Age & $62.7 \pm 7.5$ & $69.4 \pm 6.6$ & $<0.001$ & $63.0 \pm 7.5$ & $67.5 \pm 10.6$ & 0.18 & $62.9 \pm 7.5$ & $69.6 \pm 3.7$ & 0.02 \\
\hline Weight (kg) & $68.0 \pm 12.2$ & $52.9 \pm 7.6$ & $<0.001$ & $67.5 \pm 12.4$ & $67.0 \pm 11.1$ & 0.93 & $67.8 \pm 12.2$ & $46.7 \pm 3.5$ & $<0.001$ \\
\hline Height $(\mathrm{cm})$ & $162.0 \pm 6.6$ & $155.7 \pm 6.7$ & $<0.001$ & $161.8 \pm 6.7$ & $151.3 \pm 4.7$ & $<0.001$ & $161.7 \pm 6.8$ & $159.8 \pm 6.6$ & 0.45 \\
\hline BMI $\left(\mathrm{kg} / \mathrm{m}^{2}\right)$ & $26.0 \pm 4.5$ & $21.9 \pm 3.4$ & $<0.001$ & $25.8 \pm 4.6$ & $29.1 \pm 3.2$ & 0.11 & $25.9 \pm 4.5$ & $18.3 \pm 0.6$ & $<0.001$ \\
\hline $\operatorname{ALM}(\mathrm{kg})$ & $17.4 \pm 2.6$ & $13.6 \pm 1.0$ & NR & $17.3 \pm 2.6$ & $14.6 \pm 1.6$ & 0.02 & $17.3 \pm 2.6$ & $12.9 \pm 0.9$ & $<0.001$ \\
\hline ALMI $\left(\mathrm{kg} / \mathrm{m}^{2}\right)$ & $6.6 \pm 0.9$ & $5.6 \pm 0.5$ & $<0.001$ & $6.6 \pm 0.9$ & $6.3 \pm 0.3$ & 0.51 & $6.6 \pm 0.9$ & $5.0 \pm 0.3$ & NR \\
\hline ALM/BMI & $0.68 \pm 0.1$ & $0.63 \pm 0.08$ & 0.03 & $0.68 \pm 0.10$ & $0.50 \pm 0.01$ & NR & $0.68 \pm 0.10$ & $0.71 \pm 0.06$ & 0.49 \\
\hline Grip strength $(\mathrm{kg})$ & $25.0 \pm 5.4$ & $13.9 \pm 1.8$ & NR & $24.7 \pm 5.7$ & $15.3 \pm 1.0$ & NR & $24.7 \pm 5.6$ & $13.4 \pm 1.6$ & NR \\
\hline \multicolumn{10}{|c|}{ (b) Bivariate and multivariable analysis of salivary cortisol levels (nmol/l) } \\
\hline$N(\%)$ & $452(96.0)$ & $19(4.0)$ & & $466(98.9)$ & $5(1.1)$ & & $464(98.5)$ & $7(1.5)$ & \\
\hline \multicolumn{10}{|l|}{ Awakening } \\
\hline Bivariate & $18.5 \pm 9.0$ & $21.5 \pm 9.4$ & 0.21 & $18.6 \pm 9.0$ & $19.9 \pm 10.3$ & 0.74 & $18.6 \pm 9.0$ & $21.4 \pm 9.8$ & 0.47 \\
\hline Multivariate & $18.5 \pm 0.4$ & $21.3 \pm 2.3$ & 0.34 & $18.6 \pm 0.4$ & $20.0 \pm 4.0$ & 0.69 & $18.6 \pm 0.4$ & $21.0 \pm 3.8$ & 0.69 \\
\hline \multicolumn{10}{|l|}{$+30 \mathrm{~min}$} \\
\hline Bivariate & $27.2 \pm 11.9$ & $29.4 \pm 15.3$ & 0.52 & $27.3 \pm 12.0$ & $23.8 \pm 11.5$ & 0.54 & $27.3 \pm 12.1$ & $26.5 \pm 6.6$ & 0.79 \\
\hline Multivariate & $27.2 \pm 0.6$ & $29.6 \pm 3.0$ & 0.53 & $27.3 \pm 0.6$ & $24.4 \pm 5.4$ & 0.62 & $27.3 \pm 0.6$ & $26.0 \pm 5.0$ & 0.88 \\
\hline \multicolumn{10}{|l|}{$11 \mathrm{AM}$} \\
\hline Bivariate & $9.1 \pm 4.5$ & $12.6 \pm 6.9$ & 0.007 & $9.3 \pm 4.7$ & $8.8 \pm 4.8$ & 0.68 & $9.2 \pm 4.5$ & $16.9 \pm 7.6$ & $<0.001$ \\
\hline Multivariate & $9.2 \pm 0.2$ & $11.9 \pm 1.1$ & 0.05 & $9.3 \pm 0.2$ & $8.7 \pm 2.1$ & 0.65 & $9.2 \pm 0.2$ & $15.9 \pm 1.8$ & 0.005 \\
\hline \multicolumn{10}{|l|}{$8 \mathrm{PM}$} \\
\hline Bivariate & $3.2 \pm 1.9$ & $5.1 \pm 3.1$ & 0.001 & $3.2 \pm 1.9$ & $6.2 \pm 4.5$ & 0.03 & $3.2 \pm 2.0$ & $4.5 \pm 2.2$ & 0.13 \\
\hline Multivariate & $3.2 \pm 0.1$ & $5.1 \pm 0.5$ & 0.001 & $3.3 \pm 0.1$ & $6.1 \pm 0.9$ & 0.04 & $3.2 \pm 0.1$ & $4.6 \pm 0.8$ & 0.14 \\
\hline \multicolumn{10}{|l|}{ AUC } \\
\hline Bivariate & $4003 \pm 1457$ & $5047 \pm 1450$ & 0.006 & $4034 \pm 1462$ & $4689 \pm 2060$ & 0.54 & $4014 \pm 1450$ & $5866 \pm 1674$ & 0.006 \\
\hline Multivariate & $4010 \pm 74$ & $4860 \pm 387$ & 0.03 & $4035 \pm 74$ & $4630 \pm 656$ & 0.60 & $4017 \pm 73$ & $5669 \pm 608$ & 0.02 \\
\hline
\end{tabular}

Results are expressed as number (percentage), as mean \pm standard deviation (Bivariate), or as age and body mass index-adjusted mean \pm standard error (Multivariate). Between-group comparisons performed using student's $t$-test in (a) and using analysis of variance in (b)

$N$ number, $B M I$ body mass index, $A L M$ appendicular lean mass, $A L M I$ ALM index (ALM/height ${ }^{2}$ ), $N R$ not relevant (included in the definition of sarcopenia), FNIH Foundation for the National Institutes of Health Sarcopenia Project, EWGSOP2 European Working Group on Sarcopenia in Older People, revised consensus, AUC salivary cortisol diurnal Area Under the Curve value

${ }^{*} p$ values were calculated on Log-transformed values

were statistically significant only for the identification of sarcopenic participants in sc-11AM tertiles classification with EWGSOP2-ALMI and FNIH2017-ALM/BMI criteria, and in sc-8PM tertiles classification with EWGSOP2\&FNIH2014ALM and FNIH2017-ALM criteria.

\section{Relationship Between Salivary Cortisol Values and Sarcopenia Components}

GS was inversely related to sc-11AM $(r=-0.155)$, sc$8 \mathrm{PM}(r=-0.124)$, and sc-AUC $(r=-0.158$; all $p \leq 0.002)$ (Spearman correlation coefficients (Supplementary Table 4)); no relationship to salivary cortisol was observed for any ALM measure or any other parameter. The calculated correlation coefficient indicates that each $10 \mathrm{nmol} / \mathrm{l}$ increase of sc-11AM is associated with decrease of 1.758 (SE 0.472 ) $\mathrm{kg}$ of GS, and each $10 \mathrm{nmol} / \mathrm{l}$ increase sc-8PM is associated with decrease of 2.929 (SE 1.115) kg of GS.

\section{Discussion}

In this postmenopausal women population-based study, sarcopenia is associated with higher salivary cortisol at $11 \mathrm{AM}, 8 \mathrm{PM}$, and diurnal AUC. Our results suggest that increased cortisol exposure even at physiological levels 
Table 3 Characteristics of participants classified as sarcopenic by any definition

\begin{tabular}{|c|c|c|c|}
\hline & Any sarcoper & efinition & \\
\hline & None & Sarcopenic & $p$-value \\
\hline$N(\%)$ & $421(89.4)$ & $50(10.6)$ & \\
\hline Age & $62.6 \pm 7.5$ & $66.9 \pm 7.1$ & $<0.001$ \\
\hline Weight (kg) & $68.2 \pm 12.0$ & $61.2 \pm 13.4$ & $<0.001$ \\
\hline Height (cm) & $162.4 \pm 6.5$ & $155.5 \pm 6.1$ & $<0.001$ \\
\hline $\mathrm{BMI}\left(\mathrm{kg} / \mathrm{m}^{2}\right)$ & $25.9 \pm 4.5$ & $25.3 \pm 5.2$ & 0.41 \\
\hline $\operatorname{ALM}(\mathrm{kg})$ & $17.6 \pm 2.5$ & $14.4 \pm 2.0$ & NR \\
\hline $\operatorname{ALMI}\left(\mathrm{kg} / \mathrm{m}^{2}\right)$ & $6.7 \pm 0.9$ & $6.0 \pm 0.8$ & NR \\
\hline ALM/BMI & $0.69 \pm 0.10$ & $0.58 \pm 0.08$ & NR \\
\hline Grip strength (kg) & $25.6 \pm 5.1$ & $15.8 \pm 2.4$ & NR \\
\hline Salivary cortisol ( & l)* & & \\
\hline Awakening & & & \\
\hline Bivariate & $18.6 \pm 8.9$ & $18.6 \pm 9.9$ & 0.98 \\
\hline Multivariable & $18.6 \pm 0.5$ & $18.5 \pm 1.4$ & 0.88 \\
\hline$+30 \min$ & & & \\
\hline Bivariate & $27.3 \pm 12.0$ & $27.0 \pm 12.2$ & 0.95 \\
\hline Multivariable & $27.3 \pm 0.6$ & $27.2 \pm 1.9$ & 0.89 \\
\hline $11 \mathrm{AM}$ & & & \\
\hline Bivariate & $9.0 \pm 4.5$ & $11.5 \pm 5.6$ & 0.003 \\
\hline Multivariable & $9.1 \pm 0.2$ & $11.2 \pm 0.7$ & 0.01 \\
\hline $8 \mathrm{PM}$ & & & \\
\hline Bivariate & $3.2 \pm 1.9$ & $4.1 \pm 2.5$ & 0.01 \\
\hline Multivariable & $3.2 \pm 0.1$ & $4.1 \pm 0.3$ & 0.03 \\
\hline AUC & & & \\
\hline Bivariate & $3964 \pm 1440$ & $4763 \pm 1549$ & 0.002 \\
\hline Multivariable & $3974 \pm 77$ & $4670 \pm 235$ & 0.008 \\
\hline
\end{tabular}

Results are expressed as mean \pm standard deviation (Bivariate) or as age and body mass index-adjusted mean \pm standard error (Multivariate). Between-group comparisons performed using student's $t$-test or *analysis of variance

$N$ number, $B M I$ body mass index, $A L M$ appendicular lean mass, ALMI ALM index (ALM/height ${ }^{2}$ ), NR not relevant (included in the definition of sarcopenia), AUC salivary cortisol diurnal Area Under the Curve value

* $p$ values were calculated on Log-transformed values

may be associated to sarcopenia development. Moreover, there is a linear inverse relationship between both salivary cortisol values at $11 \mathrm{AM}$ and $8 \mathrm{PM}$ and grip strength.

\section{Sarcopenia Prevalence}

Sarcopenia prevalence is low in our population, 1.1-6.6\% depending on the definition used, $10.6 \%$ if all of the definitions are considered. Our rate is similar to that of some studies using DXA for muscle mass measures with or without GS $(0.9-7.9 \%$ [3]), but is lower than reported in others $(4.5-21.5 \%[32,33])$. The main difference is age, which is lower in our whole population ( $>50$ years versus $>65$ years in the latter studies $[32,33])$, but with higher proportion of sarcopenic participants in higher age tertiles. We cannot rule out higher sarcopenia prevalence in the whole cohort, as excluded participants were significantly older and had lower GS (Supplementary Table 1).

The different criteria used lead to the identification of different participants, as shown in Fig. 2, even when using the same consensus definition but with muscle mass considered as an absolute value (ALM), or corrected by BMI (ALM/ $\mathrm{BMI}$ ) or height (ALMI), as observed in previous studies [4, 6, 34]. Moreover, sarcopenic participants on ALM/BMI cut-offs do not have lower ALMI, and vice versa, and older and younger participants have similar BMI values, but lower ALM. BMI changes are probably reflecting fat mass changes in this population, and lower ALM/BMI values could identify some participants with only increased fat mass. The pertinence of one or the other adjustment is still debated $[4,6,34]$.

\section{Salivary Cortisol Values in Sarcopenic Participants}

Our study suggests that sarcopenia is associated to higher diurnal levels of salivary cortisol, and thus with blunted cortisol circadian rhythm: sarcopenic participants have higher salivary cortisol at $11 \mathrm{AM}, 8 \mathrm{PM}$, and/or AUC values, and globally more sarcopenic participants are found in the highest tertile sc-11 AM or sc-8PM. To our knowledge, this is the first study to show that this association is present regardless of the definition used, or considering all participants identified by any of them, which increases its significance.

\section{Relationship Between Salivary Cortisol Values and Sarcopenia Components}

We found no significant correlation between the different salivary cortisol values and the ALM measures, eventually due to the exclusion of a potentially relevant number of sarcopenic participants as discussed before (Supplementary Table 1). Conversely, cortisol values were inversely associated to GS measures. Thus the relationship between salivary cortisol values and sarcopenia is mainly mediated by GS in our study. Interestingly, participants identified as probably sarcopenic by the presence of only a decreased GS (EWGSOP2 definitions) did not have higher cortisol levels at any time-point, even though sarcopenic diagnosis by EWGSOP2 cut-off values are the same. We hypothesize that participants with low GS and normal ALM (probably sarcopenic) represent a different population with lower performance of other origin. To our knowledge, whether this population evolves 
Table 4 Characteristics of the participants according to 11 AM or 8 PM cortisol tertiles

\begin{tabular}{|c|c|c|c|c|c|c|c|c|}
\hline & \multicolumn{4}{|c|}{$11 \mathrm{AM}$ cortisol tertiles } & \multicolumn{4}{|c|}{8 PM cortisol tertiles } \\
\hline & Tertile 1 & Tertile 2 & Tertile 3 & $p$-value & Tertile 1 & Tertile 2 & Tertile 3 & $p$-value \\
\hline Sample size & 150 & 150 & 149 & & 148 & 148 & 148 & \\
\hline Age (years) & $61.2 \pm 6.9$ & $63.9 \pm 7.9$ & $64.0 \pm 7.5$ & 0.001 & $62.1 \pm 7.0$ & $63.1 \pm 8.3$ & $63.9 \pm 7.3$ & 0.13 \\
\hline Weight (kg) & $67.7 \pm 12.4$ & $69.2 \pm 12.7$ & $65.3 \pm 11.9$ & 0.02 & $68.5 \pm 12.8$ & $66.4 \pm 10.7$ & $67.3 \pm 13.0$ & 0.35 \\
\hline Height $(\mathrm{cm})$ & $161.8 \pm 7.1$ & $162.1 \pm 6.3$ & $161.3 \pm 6.6$ & 0.57 & $162.2 \pm 7.2$ & $162.0 \pm 6.4$ & $160.9 \pm 6.6$ & 0.22 \\
\hline $\operatorname{BMI}\left(\mathrm{kg} / \mathrm{m}^{2}\right)$ & $25.9 \pm 4.5$ & $26.4 \pm 4.6$ & $25.2 \pm 4.6$ & 0.08 & $26.0 \pm 4.5$ & $25.4 \pm 4.3$ & $26.0 \pm 4.7$ & 0.42 \\
\hline $\operatorname{ALM}(\mathrm{kg})$ & $17.4 \pm 2.7$ & $17.6 \pm 2.7$ & $16.8 \pm 2.5$ & 0.04 & $17.5 \pm 2.8$ & $17.2 \pm 2.2$ & $17.1 \pm 2.9$ & 0.49 \\
\hline ALMI $\left(\mathrm{kg} / \mathrm{m}^{2}\right)$ & $6.6 \pm 0.9$ & $6.7 \pm 0.9$ & $6.5 \pm 0.9$ & 0.08 & $6.6 \pm 0.9$ & $6.6 \pm 0.8$ & $6.6 \pm 0.9$ & 0.81 \\
\hline $\operatorname{ALM}(\mathrm{kg}) / \mathrm{BMI}\left(\mathrm{kg} / \mathrm{m}^{2}\right)$ & $0.68 \pm 0.10$ & $0.68 \pm 0.10$ & $0.68 \pm 0.10$ & 0.80 & $0.68 \pm 0.10$ & $0.69 \pm 0.10$ & $0.67 \pm 0.10$ & 0.26 \\
\hline Grip strength (kg) & $25.8 \pm 5.4$ & $24.3 \pm 5.6$ & $23.6 \pm 6.0$ & 0.005 & $25.1 \pm 5.7$ & $24.8 \pm 5.8$ & $23.8 \pm 5.8$ & 0.10 \\
\hline \multicolumn{9}{|l|}{ Sarcopenia definition $n(\%)^{\#}$} \\
\hline EWGSOP2 \& FNIH2014-ALM & $2(1.3)$ & $7(4.7)$ & $9(6.0)$ & 0.08 & $3(2.0)$ & $2(1.4)$ & $13(8.8)$ & 0.004 \\
\hline FNIH2014-ALM/BMI & $1(0.7)$ & $3(2.0)$ & $1(0.7)$ & 0.63 & $0(0)$ & $2(1.4)$ & $3(2.0)$ & 0.38 \\
\hline EWGSOP2-ALMI & $0(0.0)$ & $1(0.7)$ & $6(4.0)$ & 0.007 & $1(0.7)$ & $1(0.7)$ & $4(2.7)$ & 0.38 \\
\hline FNIH2017-ALM & $6(4.0)$ & $9(6.0)$ & $8(5.4)$ & 0.75 & $7(4.7)$ & $3(2.0)$ & $13(8.8)$ & 0.04 \\
\hline FNIH2017-ALM/BMI & $4(2.7)$ & $10(6.7)$ & $15(10.1)$ & 0.03 & $7(4.7)$ & $8(5.4)$ & $14(9.5)$ & 0.23 \\
\hline EWGSOP-ALMI & $2(1.3)$ & $4(2.7)$ & $9(6.0)$ & 0.08 & $3(2.0)$ & $2(1.4)$ & $9(6.1)$ & 0.08 \\
\hline Any definition & $7(4.7)$ & $16(10.7)$ & $24(16.2)$ & 0.004 & $12(8.1)$ & $10(6.8)$ & $25(17.0)$ & 0.01 \\
\hline
\end{tabular}

Results are expressed as mean \pm standard deviation or as number (percentage)

$B M I$ body mass index, ALM appendicular lean mass, ALMI ALM index (ALM/height ${ }^{2}$ ), FNIH Foundation for the National Institutes of Health Sarcopenia Project, EWGSOP European Working Group on Sarcopenia in Older People

Between-group comparisons performed using analysis of variance or "Fisher's exact test

toward a sarcopenia diagnosis over time due to a decrease of ALM has never been studied.

Most published studies on cortisol and muscle quantity or function have not considered free cortisol (the active form measured in saliva or urines) or its circadian rhythm. Those who analyzed the relationship between free cortisol or circadian rhythm and DXA for ALM quantification, GS for muscle function assessment, or sarcopenia diagnosis using both methods [15, 16, 18, 19, 35, 36], have produced conflicting results. Two studies found a positive association between free cortisol and GS. Heaney et al. (36 healthy participants, $72.5 \pm 6.5$ years, 18 women) [36] found that lower overall salivary cortisol levels (driven by lower early morning and 30 min after waking values) were associated with lower GS. Older participants had higher mid-day values, but were not separately analyzed. In another study with 798 all-age participants (mean age $48.6 \pm 17.3$ years, $45.1 \%$ women) [35], Bochud et al. showed a positive association between GS and total lean mass/height ${ }^{2}$ (but not ALMI) measured by DXA, and different cortisol and cortisone metabolites measured in urine; the association was not present in older participants, and the authors did not specifically analyze free cortisol. Two other studies found lower muscle function with higher evening salivary cortisol, but no relationship with GS in particular in the cross-sectional analysis [18]. In the first study (1046 participants, mean age $74.5 \pm 7.0$ years, $51.8 \%$ women) [16], during a 3-year follow-up GS loss was higher in participants with higher evening salivary cortisol values. In the second study, an individual meta-analysis of cortisol and muscle function data from several cohorts (participants mean age of 61-74 years) [18], the authors found no association between salivary cortisol and GS measures. However, only two cohorts had both measures, one with non-published data, and the previously discussed negative study [16], and higher salivary cortisol levels were associated with poorer performance in other tests of muscle function (walking speed, chair rises and standing balance) [17].

Finally, two studies showed a relationship between higher salivary cortisol and sarcopenia or muscle function [19]. In one small study ( $n=45$, mean age $77.6 \pm 6.5$ years, 21 women) [19] sarcopenic participants as defined by Baumgartner et al. [37] (on the sole basis of ALMI, with similar cut-off values as EWGSOP-ALMI definition) had higher diurnal sc-AUC than sarcopenic obese, obese, and normal patients. In a population-study including 745 participants (mean age 75.1 years, range 65-90, 363 women) [15] frail and prefrail patients had higher evening cortisol than nonfrail, which was associated to low GS and slow gait speed.

All described studies included both men and women, and either found no gender difference [16] or did not analyze data separately, which may have increased the variance of the GS or ALM measures, since men have higher values. 
In addition, there is great heterogeneity between studies in the definition of normal cortisol values: some used a cut-off (20\% of the population with lower values [15] or different published criteria $[19,36])$; others looked for linear associations of either the $z$-score [18] or absolute values [35]; one study compared values between participants with highest or lower salivary cortisol [16].

\section{Strengths and Limitations}

Main limitations of our study are due to the cross-sectional observational design. Also, we only collected one salivary cortisol probe per time-point per participant, and evening values have not been measured at midnight, as proposed for hypercortisolism screening, to facilitate compliance to saliva collection. Muscle function displays significant circadian variations [38], and GS has been measured in the morning but not at the same hour in every participant, and salivary cortisol and GS have not been measured the same day. Finally, awakening hour has not been recorded. Exclusion of participants decreases sample size and limits the generalization. However included participants had higher GS measures and had lower evening cortisol; it is probable that data of the full cohort would have yielded similar results.

On the other hand, the study has multiple strengths. The OsteoLaus cohort is a large homogeneous population sample of postmenopausal women that allows for adequate statistical power. The most accurate method for body composition assessment, DXA, has been used. Also, even though multiple association analysis have been made to characterize the population, results are similar and highly significant whatever sarcopenia definition is used, and for both $11 \mathrm{AM}$ and 8 PM time-points, and the resulting sc-AUC values.

\section{Conclusion}

Our study suggests that increased diurnal cortisol impregnation with aging is associated to sarcopenia diagnosis in postmenopausal women, and may have a role in its development. More studies are needed, mainly with longitudinal analysis, to determine whether salivary cortisol, an easy and non-stressful method of cortisol measurement, could have a clinical utility in identifying patients at risk for sarcopenia.

Supplementary Information The online version contains supplementary material available at https://doi.org/10.1007/s00223-021-00863-y.

\footnotetext{
Acknowledgements The authors would like to thank Marie Almudena Metzger, the research nurse of the OsteoLaus cohort, for data collection and management of the OsteoLaus study, and Romana Pfaffen as well as other participating radiology technicians for the performance of the body composition exams by DXA.
}

Author Contributions All authors contributed to the study conception and design. Material preparation and data collection and were performed by PM-V, BA-R, MP, CK, PV, GW, DH, and OL. PM-V and EGR performed data analysis. EGR wrote the first draft of the manuscript, and all authors commented on previous versions of the manuscript. All authors read and approved the final manuscript.

Funding Open Access funding provided by Université de Lausanne. The OsteoLaus study is supported by research grants from Lausanne University Hospital (Centre Hospitalier Universitaire Vaudois strategic plan funds) and the Swiss National Science Foundation (Grant 32473B_156978). The CoLaus/PsyCoLaus study was and is supported by research grants from GlaxoSmithKline, the Faculty of Biology and Medicine of Lausanne, and the Swiss National Science Foundation (Grants 3200B0-105993, 3200B0-118308, 33CSCO-122661, 33CS30139468, 33CS30-148401 and 32473B-182210). Dr Gerard Waeber research is supported by the Swiss National Science Foundation Grant 32003B-17309. Dr Gonzalez Rodriguez research is supported by the Faculty of Biology and Medicine of Lausanne/Lausanne University Hospital ProFemmes grant, and the Fondation Suisse contre l'Ostéoporose (Lausanne). These funding sources had no involvement in the study design, data collection, analysis and interpretation, writing of the report, or decision to submit the article for publication.

Data Availability The datasets generated during and/or analyzed during the current study are not publicly available but are available from the corresponding author on reasonable request.

Code Availability Not applicable.

\section{Declarations}

Disclosures EGR, PMV, BAR, GP, MP, CK, PV, GW, DH and OL declare that they have no conflict of interest.

Ethical Approval All procedures performed in studies involving human participants were in accordance with the ethical standards of the institutional and/or national research committee and with the 1964 Helsinki declaration and its later amendments or comparable ethical standards.

Consent to Participate Informed consent was obtained from all individual participants included in the study.

Consent for Publication Not applicable.

Open Access This article is licensed under a Creative Commons Attribution 4.0 International License, which permits use, sharing, adaptation, distribution and reproduction in any medium or format, as long as you give appropriate credit to the original author(s) and the source, provide a link to the Creative Commons licence, and indicate if changes were made. The images or other third party material in this article are included in the article's Creative Commons licence, unless indicated otherwise in a credit line to the material. If material is not included in the article's Creative Commons licence and your intended use is not permitted by statutory regulation or exceeds the permitted use, you will need to obtain permission directly from the copyright holder. To view a copy of this licence, visit http://creativecommons.org/licenses/by/4.0/. 


\section{References}

1. Cruz-Jentoft AJ, Bahat G, Bauer J, Boirie Y, Bruyere O, Cederholm T, Cooper C et al (2019) Sarcopenia: revised European consensus on definition and diagnosis. Age Ageing 48(1):16-31. https://doi.org/10.1093/ageing/afy169

2. Prado CM, Purcell SA, Alish C, Pereira SL, Deutz NE, Heyland DK, Goodpaster BH et al (2018) Implications of low muscle mass across the continuum of care: a narrative review. Ann Med 50(8):675-693. https://doi.org/10.1080/07853890.2018.1511918

3. Cruz-Jentoft AJ, Landi F, Schneider SM, Zuniga C, Arai H, Boirie Y, Chen LK et al (2014) Prevalence of and interventions for sarcopenia in ageing adults: a systematic review. Report of the International Sarcopenia Initiative (EWGSOP and IWGS). Age Ageing 43(6):748-59. https://doi.org/10.1093/ageing/afu115

4. Beaudart C, Reginster JY, Slomian J, Buckinx F, Dardenne N, Quabron A, Slangen C et al (2015) Estimation of sarcopenia prevalence using various assessment tools. Exp Gerontol 61:31-37. https://doi.org/10.1016/j.exger.2014.11.014

5. Batsis JA, Barre LK, Mackenzie TA, Pratt SI, Lopez-Jimenez F, Bartels S J (2013) Variation in the prevalence of sarcopenia and sarcopenic obesity in older adults associated with different research definitions: dual-energy X-ray absorptiometry data from the National Health and Nutrition Examination Survey 19992004. J Am Geriatr Soc 61:974

6. Bijlsma AY, Meskers CG, Ling CH, Narici M, Kurrle SE, Cameron ID, Westendorp RG et al (2013) Defining sarcopenia: the impact of different diagnostic criteria on the prevalence of sarcopenia in a large middle aged cohort. Age (Dordr) 35(3):871-881. https://doi.org/10.1007/s11357-012-9384-z

7. Cruz-Jentoft AJ, Sayer AA (2019) Sarcopenia. Lancet 393(10191):2636-2646. https://doi.org/10.1016/S0140-6736(19) 31138-9

8. Minetto MA, Lanfranco F, Motta G, Allasia S, Arvat E, D'Antona $\mathrm{G}$ (2011) Steroid myopathy: some unresolved issues. J Endocrinol Investig 34(5):370-375. https://doi.org/10.1007/BF03347462

9. Van Cauter E, Leproult R, Kupfer DJ (1996) Effects of gender and age on the levels and circadian rhythmicity of plasma cortisol. J Clin Endocrinol Metab 81(7):2468-2473. https://doi.org/10.1210/ jcem.81.7.8675562

10. Veldhuis JD, Sharma A, Roelfsema F (2013) Age-dependent and gender-dependent regulation of hypothalamic-adrenocorticotropic-adrenal axis. Endocrinol Metab Clin N Am 42(2):201-225. https://doi.org/10.1016/j.ecl.2013.02.002

11. Nater UM, Hoppmann CA, Scott SB (2013) Diurnal profiles of salivary cortisol and alpha-amylase change across the adult lifespan: evidence from repeated daily life assessments. Psychoneuroendocrinology 38(12):3167-3171. https://doi.org/10.1016/j. psyneuen.2013.09.008

12. Roubenoff R, Rall LC (1993) Humoral mediation of changing body composition during aging and chronic inflammation. Nutr Rev 51(1):1-11. https://doi.org/10.1111/j.1753-4887.1993.tb030 45. $\mathrm{x}$

13. Kim JH, Kwak MK, Ahn SH, Kim H, Cho YY, Suh S, Kim BJ et al (2018) Alteration in skeletal muscle mass in women with subclinical hypercortisolism. Endocrine 61(1):134-143. https:// doi.org/10.1007/s12020-018-1598-0

14. Moraes AB, Cavalari EMR, de Paula MP, Arruda M, Curi DSC, Leitao RA, de Mendonca LMC et al (2019) Evaluation of body composition using dual-energy $\mathrm{X}$-ray absorptiometry in patients with non-functioning adrenal incidentalomas and an intermediate phenotype: Is there an association with metabolic syndrome? J Endocrinol Investig 42(7):797-807. https://doi.org/10.1007/ s40618-018-0985-y
15. Johar H, Emeny RT, Bidlingmaier M, Reincke M, Thorand B, Peters A, Heier M et al (2014) Blunted diurnal cortisol pattern is associated with frailty: a cross-sectional study of 745 participants aged 65 to 90 years. J Clin Endocrinol Metab 99(3):E464-E468. https://doi.org/10.1210/jc.2013-3079

16. Peeters GM, van Schoor NM, van Rossum EF, Visser M, Lips P (2008) The relationship between cortisol, muscle mass and muscle strength in older persons and the role of genetic variations in the glucocorticoid receptor. Clin Endocrinol (Oxf) 69(4):673-682. https://doi.org/10.1111/j.1365-2265.2008.03212.x

17. Peeters GM, van Schoor NM, Visser M, Knol DL, Eekhoff EM, de Ronde W, Lips P (2007) Relationship between cortisol and physical performance in older persons. Clin Endocrinol (Oxf) 67(3):398-406. https://doi.org/10.1111/j.1365-2265.2007.02900.x

18. Gardner MP, Lightman S, Sayer AA, Cooper C, Cooper R, Deeg D, Ebrahim S et al (2013) Dysregulation of the hypothalamic pituitary adrenal (HPA) axis and physical performance at older ages: an individual participant meta-analysis. Psychoneuroendocrinology 38(1):40-49. https://doi.org/10.1016/j.psyneuen.2012. 04.016

19. Waters DL, Qualls CR, Dorin RI, Veldhuis JD, Baumgartner RN (2008) Altered growth hormone, cortisol, and leptin secretion in healthy elderly persons with sarcopenia and mixed body composition phenotypes. J Gerontol A Biol Sci Med Sci 63(5):536-541. https://doi.org/10.1093/gerona/63.5.536

20. Gonzalez Rodriguez E, Lamy O, Stoll D, Metzger M, Preisig M, Kuehner C, Vollenweider P et al (2017) High evening cortisol level is associated with low TBS and increased prevalent vertebral fractures: OsteoLaus study. J Clin Endocrinol Metab 102(7):2628-2636. https://doi.org/10.1210/jc.2016-3804

21. Preisig M, Waeber G, Vollenweider P, Bovet P, Rothen S, Vandeleur C, Guex P et al (2009) The PsyCoLaus study: methodology and characteristics of the sample of a population-based survey on psychiatric disorders and their association with genetic and cardiovascular risk factors. BMC Psychiatry 9:9. https://doi.org/ 10.1186/1471-244X-9-9

22. Shevroja E, Marques-Vidal P, Aubry-Rozier B, Hans G, Rivadeneira F, Lamy O, Hans D (2019) Cohort profile: the OsteoLaus study. Int J Epidemiol 48(4):1046-1047. https://doi.org/10.1093/ ije/dyy 276

23. Ouanes S, Castelao E, Gebreab S, von Gunten A, Preisig M, Popp J (2017) Life events, salivary cortisol, and cognitive performance in nondemented subjects: a population-based study. Neurobiol Aging 51:1-8. https://doi.org/10.1016/j.neurobiolaging.2016.11. 014

24. Marques-Vidal P, Bochud M, Bastardot F, von Känel R, Aubry J, Gaspoz J, Paccaud F et al (2011) Assessing the associations between mental disorders, cardiovascular risk factors, and cardiovascular disease: the CoLaus/PsyCoLaus Study. Raisons de santé [Internet]. 06.10.2016; 182. https://www.iumsp.ch/Publications/ pdf/rds182_fr.pdf

25. Kuehner C, Holzhauer S, Huffziger S (2007) Decreased cortisol response to awakening is associated with cognitive vulnerability to depression in a nonclinical sample of young adults. Psychoneuroendocrinology 32(2):199-209. https://doi.org/10.1016/j.psyne uen.2006.12.007

26. Pruessner JC, Kirschbaum C, Meinlschmid G, Hellhammer DH (2003) Two formulas for computation of the area under the curve represent measures of total hormone concentration versus timedependent change. Psychoneuroendocrinology 28(7):916-931. https://doi.org/10.1016/s0306-4530(02)00108-7

27. Petak S, Barbu CG, Yu EW, Fielding R, Mulligan K, Sabowitz $\mathrm{B}, \mathrm{Wu} \mathrm{CH}$ et al (2013) The official positions of the International Society for Clinical Densitometry: body composition analysis reporting. J Clin Densitom 16(4):508-519. https://doi.org/10. 1016/j.jocd.2013.08.018 
28. Roberts HC, Denison HJ, Martin HJ, Patel HP, Syddall H, Cooper C, Sayer AA (2011) A review of the measurement of grip strength in clinical and epidemiological studies: towards a standardised approach. Age Ageing 40(4):423-429. https://doi.org/10.1093/ ageing/afr051

29. Studenski SA, Peters KW, Alley DE, Cawthon PM, McLean RR, Harris TB, Ferrucci L et al (2014) The FNIH sarcopenia project: rationale, study description, conference recommendations, and final estimates. J Gerontol A Biol Sci Med Sci 69(5):547-558. https://doi.org/10.1093/gerona/glu010

30. Chiles Shaffer N, Ferrucci L, Shardell M, Simonsick EM, Studenski S (2017) Agreement and predictive validity using less-conservative foundation for the National Institutes of Health Sarcopenia Project Weakness Cutpoints. J Am Geriatr Soc 65(3):574-579. https://doi.org/10.1111/jgs.14706

31. Cruz-Jentoft AJ, Baeyens JP, Bauer JM, Boirie Y, Cederholm T, Landi F, Martin FC et al (2010) Sarcopenia: European consensus on definition and diagnosis: report of the European Working Group on Sarcopenia in Older People. Age Ageing 39(4):412423. https://doi.org/10.1093/ageing/afq034

32. Beaudart C, Reginster JY, Slomian J, Buckinx F, Locquet M, Bruyere O (2014) Prevalence of sarcopenia: the impact of different diagnostic cut-off limits. J Musculoskelet Neuronal Interact 14(4):425-431

33. Bischoff-Ferrari HA, Orav JE, Kanis JA, Rizzoli R, Schlogl M, Staehelin HB, Willett WC et al (2015) Comparative performance of current definitions of sarcopenia against the prospective incidence of falls among community-dwelling seniors age 65 and older. Osteoporos Int 26(12):2793-2802. https://doi.org/10.1007/ s00198-015-3194-y
34. Kim KM, Jang HC, Lim S (2016) Differences among skeletal muscle mass indices derived from height-, weight-, and body mass index-adjusted models in assessing sarcopenia. Korean J Intern Med 31(4):643-650. https://doi.org/10.3904/kjim.2016.015

35. Bochud M, Ponte B, Pruijm M, Ackermann D, Guessous I, Ehret G, Escher G et al (2019) Urinary sex steroid and glucocorticoid hormones are associated with muscle mass and strength in healthy adults. J Clin Endocrinol Metab 104(6):2195-2215. https://doi. org/10.1210/jc.2018-01942

36. Heaney JL, Phillips AC, Carroll D (2012) Ageing, physical function, and the diurnal rhythms of cortisol and dehydroepiandrosterone. Psychoneuroendocrinology 37(3):341-349. https://doi.org/ 10.1016/j.psyneuen.2011.07.001

37. Baumgartner RN, Koehler KM, Gallagher D, Romero L, Heymsfield SB, Ross RR, Garry PJ et al (1998) Epidemiology of sarcopenia among the elderly in New Mexico. Am J Epidemiol 147(8):755-763. https://doi.org/10.1093/oxfordjournals.aje.a0095 20

38. Vitale JA, Bonato M, La Torre A, Banfi G (2019) The role of the molecular clock in promoting skeletal muscle growth and protecting against sarcopenia. Int J Mol Sci 20(17):4318. https://doi.org/ 10.3390/ijms20174318

Publisher's Note Springer Nature remains neutral with regard to jurisdictional claims in published maps and institutional affiliations. 